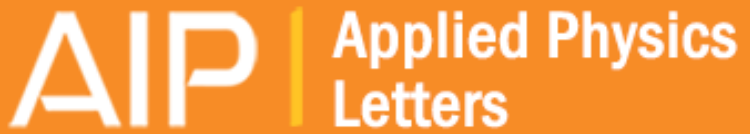

\section{Angle-resolved photoemission spectroscopy with 9-eV photon-energy pulses generated in a gas-filled hollow-core photonic crystal fiber \\ H. Bromberger, A. Ermolov, F. Belli, H. Liu, F. Calegari, M. Chávez-Cervantes, M. T. Li, C. T. Lin, A. Abdolvand, P. St. J. Russell, A. Cavalleri, J. C. Travers, and I. Gierz}

Citation: Applied Physics Letters 107, 091101 (2015); doi: 10.1063/1.4929542

View online: http://dx.doi.org/10.1063/1.4929542

View Table of Contents: http://scitation.aip.org/content/aip/journal/apl/107/9?ver=pdfcov

Published by the AIP Publishing

\section{Articles you may be interested in}

Fluorescence-based remote irradiation sensor in liquid-filled hollow-core photonic crystal fiber

Appl. Phys. Lett. 108, 231107 (2016); 10.1063/1.4953590

Quasiparticle dynamics across the full Brillouin zone of $\mathrm{Bi} 2 \mathrm{Sr} 2 \mathrm{CaCu} 2 \mathrm{O} 8+\delta$ traced with ultrafast time and angleresolved photoemission spectroscopy

Struct. Dyn. 2, 054501 (2015); 10.1063/1.4933133

Preparation of layered thin film samples for angle-resolved photoemission spectroscopy

Appl. Phys. Lett. 105, 121608 (2014); 10.1063/1.4896632

Plasma-induced nonlinear optical phenomena in gas-filled hollow-core PCFs

AIP Conf. Proc. 1475, 6 (2012); 10.1063/1.4750077

APL Photonics

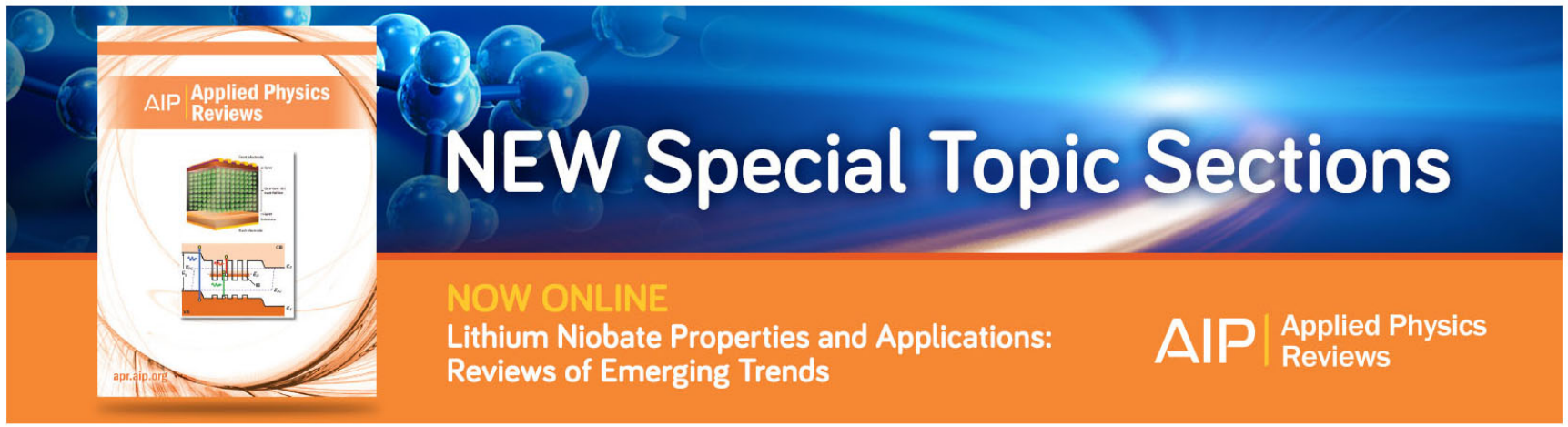




\title{
Angle-resolved photoemission spectroscopy with 9-eV photon-energy pulses generated in a gas-filled hollow-core photonic crystal fiber
}

\author{
H. Bromberger, ${ }^{1, a)}$ A. Ermolov, ${ }^{2}$ F. Belli, ${ }^{2}$ H. Liu, ${ }^{1}$ F. Calegari, ${ }^{1,3}$ M. Chávez-Cervantes, ${ }^{1}$ \\ M. T. Li, ${ }^{4}$ C. T. Lin, ${ }^{4}$ A. Abdolvand, ${ }^{2}$ P. St. J. Russell, ${ }^{2}$ A. Cavalleri, ${ }^{1,5}$ J. C. Travers, ${ }^{2}$ \\ and I. Gierz ${ }^{1}$ \\ ${ }^{1}$ Max Planck Institute for the Structure and Dynamics of Matter, Luruper Chaussee 149, 22761 Hamburg, \\ Germany \\ ${ }^{2}$ Max Planck Institute for the Science of Light, Günther-Scharowsky-Str. 1, 91058 Erlangen, Germany \\ ${ }^{3}$ Institute for Photonics and Nanotechnologies, IFN-CNR, Piazza Leonardo da Vinci 32, I-20133 Milano, Italy \\ ${ }^{4}$ Max Planck Institute for Solid State Research, Heisenbergstr. 1, 70569 Stuttgart, Germany \\ ${ }^{5}$ Clarendon Laboratory, Department of Physics, University of Oxford, Parks Rd. Oxford OX1 3PU, \\ United Kingdom
}

(Received 27 April 2015; accepted 12 July 2015; published online 31 August 2015)

\begin{abstract}
A recently developed source of ultraviolet radiation, based on optical soliton propagation in a gasfilled hollow-core photonic crystal fiber, is applied here to angle-resolved photoemission spectroscopy (ARPES). Near-infrared femtosecond pulses of only few $\mu \mathrm{J}$ energy generate vacuum ultraviolet radiation between 5.5 and $9 \mathrm{eV}$ inside the gas-filled fiber. These pulses are used to measure the band structure of the topological insulator $\mathrm{Bi}_{2} \mathrm{Se}_{3}$ with a signal to noise ratio comparable to that obtained with high order harmonics from a gas jet. The two-order-of-magnitude gain in efficiency promises time-resolved ARPES measurements at repetition rates of hundreds of $\mathrm{kHz}$ or even $\mathrm{MHz}$, with photon energies that cover the first Brillouin zone of most materials. (C) 2015 AIP Publishing LLC.

[http://dx.doi.org/10.1063/1.4929542]
\end{abstract}

The photoelectric effect ${ }^{1}$ occurs only if a solid is irradiated with photon energies in excess of its work function, which typically lies between 4.5 and $4.8 \mathrm{eV} .^{2}$ By measuring the kinetic energy and emission angle of the generated photoelectrons with angle-resolved photoemission spectroscopy (ARPES), the binding energy, $\mathrm{E}_{\mathrm{B}}$, and the in-plane momentum, $\mathrm{k}_{\|}$, of the electrons inside the crystal can be determined. Thus, the technique yields the spectral function of the solid and has evolved into an extraordinarily powerful tool for condensed matter research. ${ }^{2}$

The most commonly used light sources for static ARPES are either monochromatized helium lamps (He I $\alpha$ line at $21 \mathrm{eV}$ and $\mathrm{He} \mathrm{II} \alpha$ line at $41 \mathrm{eV}$ ) or synchrotron sources, where linewidths below $1 \mathrm{meV}$ have been possible. The typical energy and angle resolution of state-of-the-art analyzers are $\Delta \mathrm{E}<10 \mathrm{meV}$ and $\Delta \Theta=0.1^{\circ}$. In the ARPES measurement, both the accessible momentum resolution, $\Delta \mathrm{k}_{\|}$, and maximum in-plane momentum, $\mathrm{k}_{\max }$, depend on the photon energy. The momentum resolution is thus higher at low photon energies, as shown in recent experiments with $6 \mathrm{eV}$ photons from a continuous wave laser-based ARPES setup, which yielded momentum resolutions as low as $\Delta \mathrm{k}_{\|}$ $=0.006 \AA^{-1}$.

Pulsed sources are attractive because they can be used for time-resolved ARPES (tr-ARPES) experiments. ${ }^{4-7}$ Tr-ARPES probes are often based on the fourth harmonic of femtosecond Ti:sapphire (TiSa) lasers. ${ }^{8}$ This process has a conversion efficiency of a few percent, resulting in excellent signal-to-noise ratios due to the high repetition rate of the laser source. However, absorption in the nonlinear crystal (e.g., in beta barium borate- $\mathrm{BBO}$ ) limits the maximum photon energy range

${ }^{\text {a)} H u b e r t u s . B r o m b e r g e r @ m p s d . m p g . d e ~}$ to $\leq 6 \mathrm{eV}$ and restricts the accessible momentum range in an ARPES experiment to $\mathrm{k}_{\max } \leq 0.6 \AA^{-1}$.

Recently, Liu et al. increased the accessible momentum range to $\mathrm{k}_{\max }=0.8 \AA^{-1}$ by frequency doubling a frequency tripled commercial $\mathrm{Nd}: \mathrm{YVO}_{4}$ laser in a potassium beryllium fluoroborate $(\mathrm{KBBF})$ crystal, resulting in vacuum ultraviolet (VUV) photon energies of $7 \mathrm{eV}(177 \mathrm{~nm}) .{ }^{9}$ However, the long pulse duration of $10 \mathrm{ps}$ did not allow for time-resolved studies of electron dynamics.

Higher photon energies can be generated by high harmonic generation (HHG) in noble gases pumped by amplified femtosecond lasers. HHG can yield extreme ultraviolet (XUV) radiation over a broad spectrum, extending up to several hundred $\mathrm{eV} .{ }^{10-12}$ The low conversion efficiency of this process (typically in the range $10^{-6}$ to $10^{-5}$ ) demands, however, high energy femtosecond pulses, which restricts the repetition rate of the laser system to a few $\mathrm{kHz}$ and results in comparatively low signal-to-noise ratios for space-charge limited photoemission. Furthermore, in this energy range, the momentum resolution is limited and the need for monochromatization further reduces the photon flux.

It has recently been shown that gas-filled, hollow-core photonic crystal fibers with a kagomé pattern (kagomé$\mathrm{PCF})^{13}$ can generate and guide light in the vacuum ultraviolet. ${ }^{14}$ By filling the fiber with different gas species at a suitable pressure, the group velocity dispersion at the near infrared pump wavelength of $800 \mathrm{~nm}$ can be adjusted to be anomalous while maintaining sufficient nonlinearity for the formation of high-order optical solitons, tens of femtoseconds in duration with energies of a few $\mu \mathrm{J}$. The dynamics of the resulting propagation can be optimized either for generation of a supercontinuum extending from the VUV to the infrared ${ }^{14,15}$ or for emission of femtosecond duration dispersive wave (DW) 
pulses, wavelength-tunable between $3 \mathrm{eV}(400 \mathrm{~nm})$ and $11 \mathrm{eV}$ $(113 \mathrm{~nm}) .^{13,15}$ In particular, DW pulses in the VUV (at around $8 \mathrm{eV}$ ) with energies in excess of $50 \mathrm{~nJ}$, representing $\sim 1 \%$ of the energy in the pump pulses, have been demonstrated. ${ }^{15}$

In this letter, we show that these DW pulses have sufficient photon flux, signal-to-noise ratio and stability to fulfill the requirements of tr-ARPES studies, and illustrate this by band structure measurements on the topological insulator $\mathrm{Bi}_{2} \mathrm{Se}_{3}$. Importantly, this generation scheme combines the high efficiency of solid-state nonlinear crystals with the spectral coverage of gas sources, which will make it possible to perform photoemission spectroscopy at photon energies that cover the whole Brillouin zone of most materials with high repetition rates.

Figure 1 shows a sketch of the experimental apparatus. We used a commercial $1 \mathrm{kHz}, 30$ fs multi-pass TiSa amplifier spectrally centered at $1.55 \mathrm{eV}(800 \mathrm{~nm})$, continuously attenuated between 0.5 and $7 \mu \mathrm{J}$ with a half-wave plate (HWP) and a glass wedge (GW). The pulse duration and linear chirp were optimized using a grating compressor (not shown). The beam pointing was stabilized with an active steering mirror (ASM) and a quadrant photodiode detector. The spatial mode distribution of the laser beam was filtered by focusing into a $150 \mu \mathrm{m}$ diameter pinhole, after which it was imaged into the 28 or $34 \mu \mathrm{m}$ diameter kagomé-PCF. The coupling efficiency was always above $80 \%$. The entrance and exit faces of the fiber were located in two separate cells as in previous experiments. ${ }^{14,15}$ The light passed through a fused silica window at the entrance and a $\mathrm{MgF}_{2}$ window at the exit. Neon or hydrogen was pumped into the exit cell, and the pressure in the fiber could be adjusted up to a maximum of 30 bar. The front gas cell was evacuated down to mbar pressure so as to ensure stable launching of light into the kagomé-PCF core. The propagation loss of the kagomé-

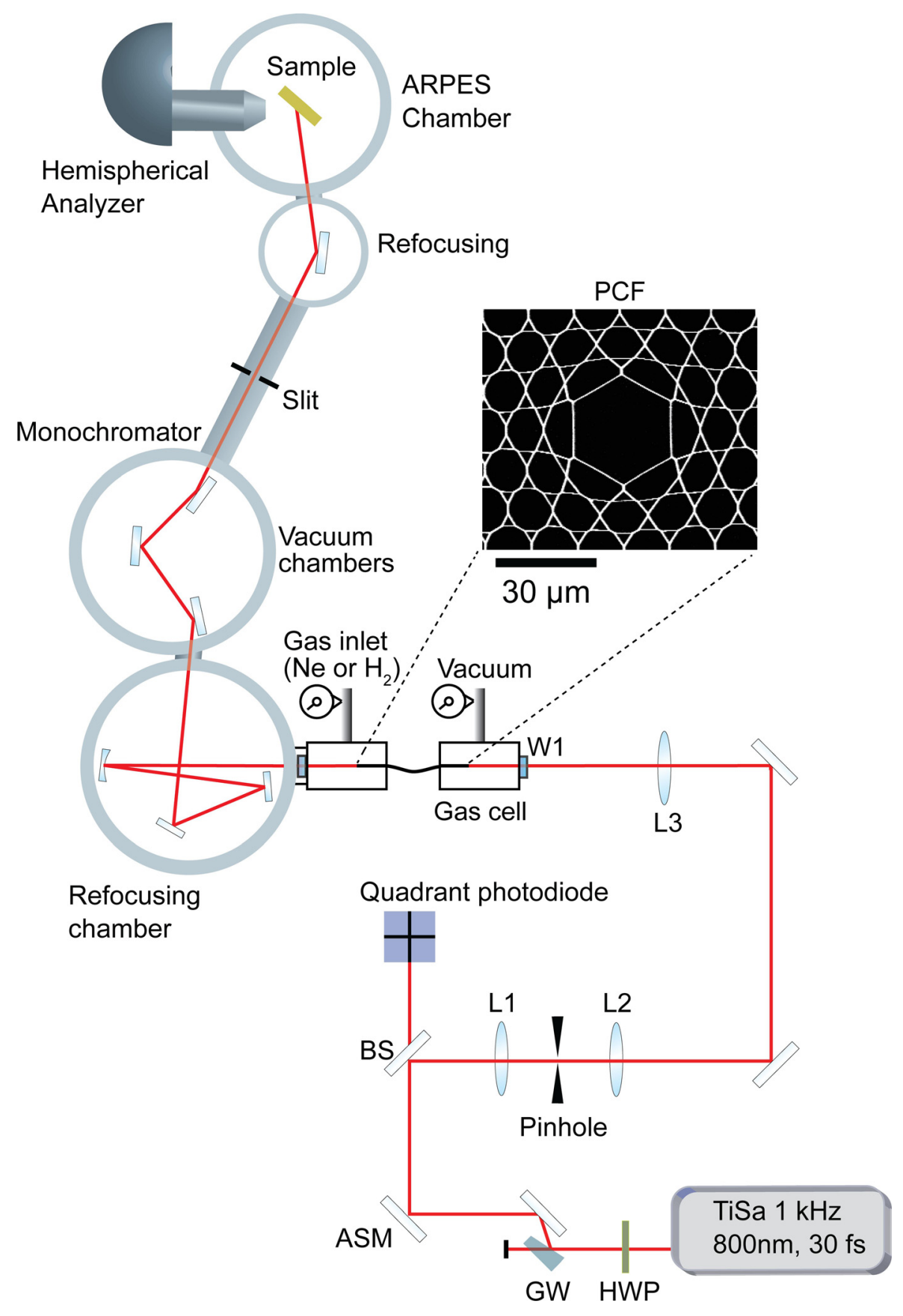

FIG. 1. Schematic of the setup. Laser light is generated in a TiSa amplifier with $1 \mathrm{kHz}$ repetition rate. A fraction of the light is split off and attenuated to below $10 \mu \mathrm{J}$ using a half-wave plate (HWP) and a glass wedge (GW). Beam-pointing stability is achieved using an active steering mirror (ASM) with a quadrant photodiode. The beam profile is cleaned by focusing the beam (with lens L1) onto a pinhole and then re-collimating it using a second lens (L2). Using a suitable chosen third lens (L3), the beam is then launched into the core $(28 \mu \mathrm{m}$ or $34 \mu \mathrm{m}$ in diameter) of the kagomé-PCF, which is held between two gas-cells. A positive pressure gradient from vacuum to the set pressure is maintained between the gas-cells. Inside the refocusing chamber, the divergent UV light is refocused and steered into the monochromator chamber, where a single grating is used for wavelength selection. Finally, the UV light is focused onto the sample inside the ARPES chamber with a toroidal mirror. Photoelectrons are dispersed with a hemispherical analyzer and counted on a two-dimensional detector. 
$\mathrm{PCF}$ is $\sim 1 \mathrm{~dB} / \mathrm{m}$ around $800 \mathrm{~nm}$. In the deep- $\mathrm{UV}$ region $(<300 \mathrm{~nm})$, losses of $\sim 1-3 \mathrm{~dB} / \mathrm{m}$ have previously been measured, ${ }^{16,17}$ but they are unknown in the VUV region. Nevertheless, as the VUV emission is generated close to the end of the fiber, the loss is expected to play a minor role. The generated UV light was expected to be in the fundamental mode. Previous mode measurements at $270 \mathrm{~nm}$ have confirmed this, ${ }^{16,17}$ and from a theoretical perspective, the VUV light is predemoninatly emitted in the fundamental mode. The UV emission was therefore estimated to have a divergence of $\sim 10 \mathrm{mrad}$ at the exit from the fiber, and was focused on to the image point of a monochromator used to separate the UV signal from the IR pump light. The first toroidal mirror inside the monochromator collimated the light, after which it was diffracted and dispersed by a grating. The second toroidal mirror focused the dispersed light on to the exit slit, forming an imaging spectrometer.

To characterize the UV signal, we used a calibrated channel electron multiplier (CEM) to measure the photon flux as a function of grating angle (i.e., wavelength). A last toroidal mirror focused the light onto the sample. The emitted photoelectrons were then dispersed according to their kinetic energy and emission angle using a hemispherical analyzer (SPECS, Phoibos 150) and counted on a twodimensional detector array.

Figure 2 shows a representative selection of output spectra generated in the PCF, recorded with the CEM as a function of wavelength. In Fig. 2(a), the spectrum, generated in a PCF
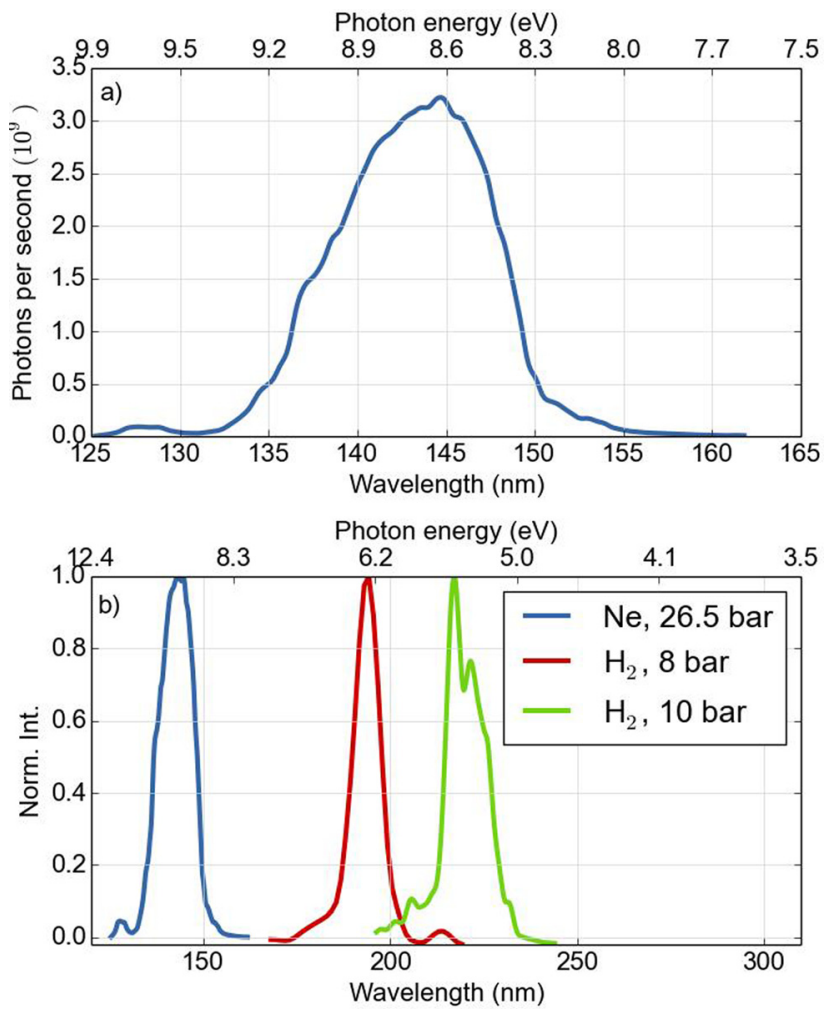

FIG. 2. UV spectra emitted by gas-filled kagomé-PCF. (a) Typical spectrum generated in 26.5 bar Ne, after the monochromator, recorded with a calibrated channel electron multiplier. The count rate is comparable to that usually observed in HHG. ${ }^{18}$ (b) Normalized spectra at different photon energies, demonstrating the tunability of the source with positive pressure gradient from low vacuum to 26.5 bar of $\mathrm{Ne}$ (blue), 8 bar of $\mathrm{H}_{2}$ (red), and 10 bar of $\mathrm{H}_{2}$ (green). filled with 26.5 bar $\mathrm{Ne}$, is centered at $8.6 \mathrm{eV}(145 \mathrm{~nm})$ with a full-width-at-half-maximum (FWHM) of $600 \mathrm{meV}(10 \mathrm{~nm})$. The photon flux of $10^{6}$ photons per pulse $\left(10^{9}\right.$ photons per second) is comparable to typical monochromatized HHG sources, ${ }^{18}$ with the important difference that $\mathrm{HHG}$ requires input pulse energies that are three orders of magnitude higher. Note that the photon flux exiting the fiber is expected to be significantly higher (based on previous measurements, more than $10^{10}$ photons per pulse ${ }^{15}$ ) but is reduced by transmission through the monochromator and refocusing optics. In Fig. 2(b), we show that the spectrum can be tuned from $5.5 \mathrm{eV}$ $(220 \mathrm{~nm})$ to $9 \mathrm{eV}(140 \mathrm{~nm})$ by changing from $\mathrm{H}_{2}$ to $\mathrm{Ne}$ and adjusting gas pressure and pulse energy. Note that, if $\mathrm{He}, \mathrm{Ar}$, and $\mathrm{Kr}$ are used in addition, continuous tuning from $11 \mathrm{eV}$ $(113 \mathrm{~nm})$ to $3 \mathrm{eV}(400 \mathrm{~nm})$ is possible. ${ }^{13-15}$ While direct VUV pulse duration measurements have so far not been performed, rigorous numerical modeling-which has been confirmed to produce excellent agreement with previous experiments, ${ }^{13-15}$ and closely reproduces the experimental spectral dynamicssuggests that the VUV radiation corresponds to coherent few femtosecond duration pulses.

To test the suitability of the PCF source for tr-ARPES studies, we performed a proof-of-principle band structure measurement on the topological insulator $\mathrm{Bi}_{2} \mathrm{Se}_{3} .{ }^{19}$ The sample consisted of stacks of Se-Bi-Se-Bi-Se quintuple layers than could be easily cleaved in situ in ultra-high vacuum. The photon energy used for ejecting the photoelectrons was set to $8.6 \mathrm{eV}(145 \mathrm{~nm})$. Figure 3 shows the measured photocurrent as a function of binding energy and in-plane momentum, revealing the typical Dirac-cone-like dispersion of quasiparticles at the surface of $\mathrm{Bi}_{2} \mathrm{Se}_{3}$ (dashed red lines are guides to the eye). The states at higher binding energy come from the bulk valence band of $\mathrm{Bi}_{2} \mathrm{Se}_{3}$. The signal-to-noise ratio of the measurement is comparable to that of other $1 \mathrm{kHz}$

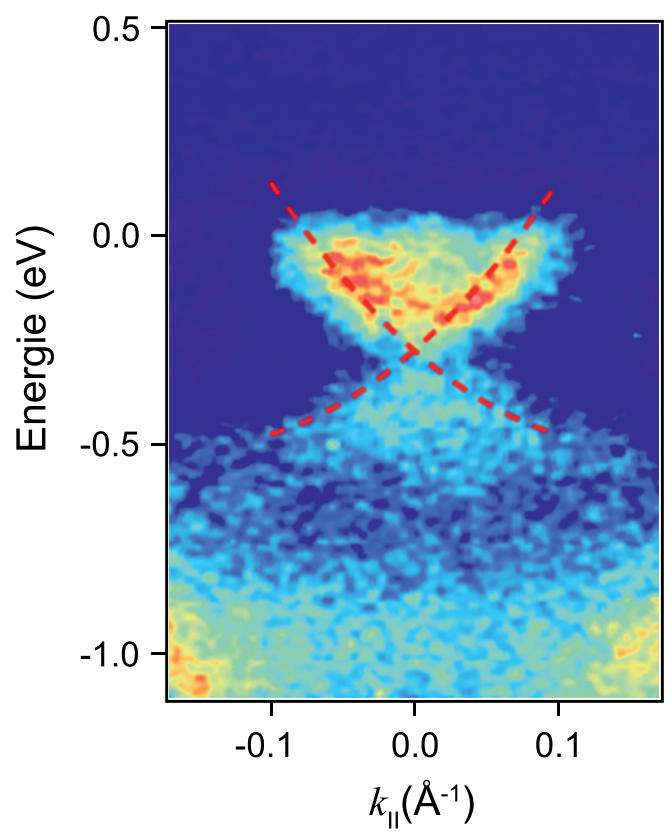

FIG. 3. ARPES spectrum of $\mathrm{Bi}_{2} \mathrm{Se}_{3}$, centered around the $\Gamma$-point of the hexagonal Brillouin zone. Dashed red lines following the surface state dispersion are guides to the eye. 
sources such as HHG, indicating that the PCF source fulfills all the requirements of tr-ARPES studies.

To summarize, we have applied the recently demonstrated VUV pulsed light source, based on dispersive-wave emission from solitons in a gas-filled kagomé-PCF, ${ }^{14,15}$ to ARPES. This source has high conversion efficiency and can be driven with pulses of only a few $\mu \mathrm{J}$ energy, making it possible to perform measurements at repetition rates of hundreds of $\mathrm{kHz}$ using existing commercial pulsed lasers. The source extends the accessible photon energy range beyond what is possible with nonlinear crystals, while retaining momentum resolution and long photoelectron escape depths characteristic of low photon energies. The source is easily tunable between 5.5 and $9 \mathrm{eV}$ with photon fluxes comparable to existing HHG sources that require three orders of magnitude more input pulse energy. The VUV spectrum can be extended out to $11 \mathrm{eV}$ by changing the filling gas to helium. The spectral bandwidth of $600 \mathrm{meV}$ theoretically provides a 3 fs transform-limited pulse, with potential for tr-ARPES studies at extreme time-scales.

We thank Jörg Harms for assisting with the figures.

${ }^{1}$ H. Hertz, Ann. Phys. 267, 983 (1887).

${ }^{2}$ S. Hüfner, Photoelectron Spectroscopy. Principles and Applications (Springer-Verlag, Berlin-Heidelberg-New York, 2003).

${ }^{3}$ A. Tamai, W. Meevasana, P. D. C. King, C. W. Nicholson, A. de la Torre, E. Rozbicki, and F. Baumberger, Phys. Rev. B 87, 075113 (2013).

${ }^{4}$ F. Schmitt, P. S. Kirchmann, U. Bovensiepen, R. G. Moore, L. Rettig, M. Krenz, J.-H. Chu, N. Ru, L. Perfetti, D. H. Lu, M. Wolf, I. R. Fisher, and Z.-X. Shen, Science 321, 1649 (2008).
${ }^{5}$ J. Graf, C. Jozwiak, C. L. Smallwood, H. Eisaki, R. A. Kaindl, D.-H. Lee, and A. Lanzara, Nat. Phys. 7, 805 (2011).

${ }^{6}$ T. Rohwer, S. Hellmann, M. Wiesenmayer, C. Sohrt, A. Stange, B. Slomski, A. Carr, Y. Liu, L. Miaja Avila, M. Kalläne, S. Mathias, L. Kipp, K. Rossnagel, and M. Bauer, Nature 471, 490 (2011).

${ }^{7}$ J. C. Petersen, S. Kaiser, N. Dean, A. Simoncig, H. Y. Liu, A. L. Cavalieri, C. Cacho, I. C. E. Turcu, E. Springate, F. Frassetto, L. Poletto, S. S. Dhesi, H. Berger, and A. Cavalleri, Phys. Rev. Lett. 107, 177402 (2011).

${ }^{8}$ J. Faure, J. Mauchain, E. Papalazarou, W. Yan, J. Pinon, M. Marsi, and L. Perfetti, Rev. Sci. Instrum. 83, 043109 (2012).

${ }^{9}$ G. Liu, G. Wang, Y. Zhu, H. Zhang, G. Zhang, X. Wang, Y. Zhou, W. Zhang, H. Liu, L. Zhao, J. Meng, X. Dong, C. Chen, Z. Xu, and X. J. Zhou, Rev. Sci. Instrum. 79, 023105 (2008).

${ }^{10}$ A. McPherson, G. Gibson, H. Jara, U. Johann, T. S. Luk, I. A. McIntyre, K. Boyer, and C. K. Rhodes, J. Opt. Soc. Am. B 4, 595 (1987).

${ }^{11}$ Ch. Spielmann, N. H. Burnett, S. Sartania, R. Koppitsch, M. Schnürer, C. Kan, M. Lenzner, P. Wobrauschek, and F. Krausz, Science 278, 661 (1997).

${ }^{12}$ A. Rundquist, C. G. Durfee III, Z. Chang, C. Herne, S. Backus, M. M. Murnane, and H. C. Kapteyn, Science 280, 1412 (1998).

${ }^{13}$ P. St., J. Russell, P. Hölzer, W. Chang, A. Abdolvand, and J. C. Travers, Nat. Photonics 8, 278-286 (2014).

${ }^{14}$ F. Belli, A. Abdolvand, W. Chang, J. C. Travers, P. St., and J. Russell, Optica 2, 292-300 (2015).

${ }^{15}$ A. Ermolov, K. F. Mak, M. H. Frosz, J. C. Travers, and P. St. J. Russell, preprint arXiv:150309033 [physics] (2015).

${ }^{16}$ N. Y. Joly, J. Nold, W. Chang, P. Hölzer, A. Nazarkin, G. K. L. Wong, F. Biancalana, and P. S. J. Russell, Phys. Rev. Lett. 106(20), 203901 (2011).

${ }^{17}$ F. Gebert, M. H. Frosz, T. Weiss, Y. Wan, A. Ermolov, N. Y. Joly, P. O. Schmidt, and P. S. J. Russell, Opt. Express 22(13), 15388 (2014).

${ }^{18}$ F. Frassetto, C. Cacho, C. A. Froud, I. C. E. Turcu, P. Villoresi, W. A. Bryan, E. Springate, and L. Poletto, Opt. Express 19(20), 19169-19181 (2011).

${ }^{19}$ D. Hsieh, Y. Xia, D. Qian, L. Wray, J. H. Dil, F. Meier, J. Osterwalder, L. Patthey, J. G. Checkelsky, N. P. Ong, A. V. Fedorov, H. Lin, A. Bansil, D. Grauer, Y. S. Hor, R. J. Cava, and M. Z. Hasan, Nature 460(7259), 1101-1105 (2009). 